\title{
DETERMINISTIC AND STOCHASTIC SIMULATIONS OF SIMPLE GENETIC CIRCUITS
}

\author{
OFER BIHAM, NATHALIE Q. BALABAN, ADIEL LOINGER \\ Racah Institute of Physics, The Hebrew University, Jerusalem 91904, Israel \\ E-mail: biham@phys.huji.ac.il,nathalieqb@phys.huji.ac.il,adiel@phys.huji.ac.il \\ AZI LIPSHTAT \\ Department of Pharmacology and Biological Chemistry, Mount Sinai \\ School of Medicine, New York, NY 10029, U.S.A. \\ E-mail: azi.lipshtat@mssm.edu \\ HAGAI B. PERETS \\ Faculty of Physics, Weizmann Institute of Science, Rehovot 76100, Israel \\ E-mail: hagai.perets@weizmann.ac.il
}

\begin{abstract}
We analyze three simple genetic circuits which involve transcriptional regulation and feedback: the autorepressor, the switch and the repressilator, that consist of one, two and three genes, respectively. Such systems are commonly simulated using rate equations, that account for the concentrations of the mRNAs and proteins produced by these genes. Rate equations are suitable when the concentrations of the relevant molecules in a cell are large and fluctuations are negligible. However, when some of the proteins in the circuit appear in low copy numbers, fluctuations become important and the rate equations fail. In this case stochastic methods, such as direct numerical integration of the master equation or Monte Carlo simulations are required. Here we present deterministic and stochastic simulations of the autorepressor, the switch and the repressilator. We show that fluctuations give rise to quantitative and qualitative changes in the dynamics of these systems. In particular, we demonstrate a fluctuations-induced bistability in a variant of the genetic switch and and noisy oscillations obtained in the repressilator circuit.
\end{abstract}

2000 Mathematics Subject Classification: Primary 92D10.

Key words and phrases: systems biology, genetic networks, genetic switch, feedback loop, stochastic analysis.

This work was supported by the Center for Complexity Studies.

The paper is in final form and no version of it will be published elsewhere. 
1. Introduction. The production of proteins in cells is regulated by networks of interacting genes. Some of these genes code for transcription factors, which are proteins that regulate the expression of other genes by binding to specific promoter sites on the DNA. Some of these proteins, called repressors, perform negative regulation, while others, called activators, perform positive regulation. Post-trancriptional regulation can occur by translational regulation, or by post-translational regulation such as protein-protein interaction, which may modify the function of these proteins. These networks of interacting genes are at the heart of all processes in cells: from the regulation of the cell cycle to the various stress responses.

It turns out that genetic networks are typically sparse networks, namely most genes interact only with a small number of other genes. Also, the networks exhibit some degree of modularity, namely one can identify recurring modules, which are known as network motifs [18].

Modules found in nature are often hard to study and fully control. To overcome these limitations, synthetic networks can be constructed from well-studied components. Examples of such components are the lactose, the lambda and the tetracycline systems in bacteria. The synthetic networks are designed to perform desired functions, determined by their architecture. They do not require the manipulation of the structure of proteins and other regulatory elements at the molecular level. The genes and promoters are often inserted into plasmids rather than on the chromosome. Two important examples of synthetic circuits are the genetic toggle switch and the repressilator.

The toggle switch consists of two genes, which negatively regulate each other expression. The regulation is performed at the transcriptional level, namely each gene codes for a repressor protein that binds to the promoter of the other gene. Such system may exhibit bistability, namely two stable states, where in each state one of the proteins is dominant and the other is suppressed. A synthetic toggle switch was constructed in $E$. coli and the conditions for bistability were examined [6]. The switching between its two states was demonstrated using chemical and thermal induction. More recently, such circuit was found to exist in a natural system in which two mutual repressors regulate the differentiation of myeloid progenitors into either macrophages or neutrophils [11].

The synthetic repressilator circuit, constructed in E. coli, was designed to exhibit oscillations, reminiscent of natural genetic oscillators such as the circadian rhythms [4]. The repressilator circuit was encoded on a plasmid that appears in a low copy number. The protein concentrations were measured vs. time in single cells. Oscillations with a period of about 150 minutes were found, namely extending over several division cycles. The oscillations were found to be noisy, typically maintaining phase coherence for times of the order of a single oscillation period.

The dynamics of genetic networks is often simulated using rate equation models. These are sets of coupled ordinary differential equations, which account for the concentrations of the mRNAs and proteins in the network. In general, rate equations account for average concentrations and ignore fluctuations. They are suitable for systems in which the concentration of interacting molecules are large and fluctuations are negligible. However, proteins in cells often appear in low copy numbers and may exhibit large fluctuations. 
Moreover, in case of transcriptional regulation, the expression of the regulated gene may be controlled by a single protein that binds to its promoter. This extends the notion of low copy numbers and the resulting fluctuations even to the case when there is a large number of free repressors of a certain type, since only one of them may bind to the promoter at any given time. Recent advances measurement techniques made it possible to measure the fluctuations in copy number of proteins in single cells [5, 23, 26]. Measurements of protein levels in single cells revealed distributions that depend on the topology of the regulatory network controlling the particular protein [19].

To account for the fluctuations, simulations of genetic networks should be done using stochastic methods such as the master equation [10, 16, 17, 20, 21, 22, 23] or Monte Carlo simulations [7, 9, 16]. The master equation provides the probability distribution of the concentrations of proteins in a population of cells. From this distribution one can calculate the average concentrations as well as the correlations and the rates of all processes. Monte Carlo simulations enable to follow the temporal variations in the level of gene expression and in the concentrations of proteins in a single cell. These results enable to extract the noise level and temporal correlation functions. They can also be used to characterize the dynamics of the system and determine whether it exhibits a single steady state, multi-stability or oscillatory behavior.

(a)

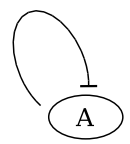

(b)

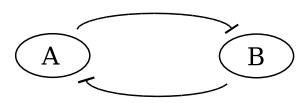

(c)

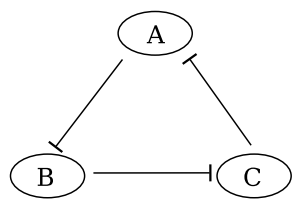

Fig. 1. The three genetic circuits considered in this paper: the autorepressor (a); the genetic switch (b); and the repressillator (c). The flat-headed arrows denote negative transcriptional regulation.

In this paper we present deterministic analysis (using rate equations) and stochastic analysis (using direct numerical integration of the master equation and Monte Carlo simulations) of three simple genetic circuits: the autorepressor, the toggle switch and the repressilator (Fig. 1). We show that fluctuations give rise to both quantitative and qualitative effect in the dynamics of thse circuits. 
The paper is organized as follows. In Sec. 2 we consider the autorepressor and present the deterministic and stochastic methods used in the paper. In Sec. 3 we study the genetic switch and in Sec. 4 we analyze the repressillator circuit. The results are discussed and summarized in Sec. 5 .

2. The autorepressor. The autorepressor is the simplest genetic circuit that involves feedback. It consist of a single gene, denoted by $a$, that negatively regulates its own expression. The gene transcribes into mRNAs that translate into $A$ proteins. These proteins function as repressors. When an $A$ protein binds to the $a$ promoter site, it prevents the RNA polymerase from binding to the promoter and thus inhibits the transcription process. It turns out that genetic networks include a large number of autorepressor modules. This circuit is thus considered as a network motif [25]. One may speculate that it performs some crucial function in the cell. It was proposed that the role of the autorepressor is to speed up response times [25] or to reduce fluctuations [2]. Below we analyze the autorepressor using deterministic and stochastic methods. We utilize its simplicity in order to present the methodologies in details.

2.1. Michaelis-Menten equations. The dynamics of genetic networks are commonly described using the Michaelis-Menten equations. These equations describe the temporal variations in the concentrations of the relevant molecules in the cell. Here, we denote the concentration of protein $A$ in a cell by $[A]$ (by concentration we refer to the average copy number of $A$ proteins per cell). The concentration of the corresponding mRNA is denoted by $[m]$. The Michaelis-Menten equations for the concentrations take the form

$$
\begin{aligned}
{[\dot{m}] } & =\frac{g_{m}}{1+k[A]^{n}}-d_{m}[m], \\
{[\dot{A}] } & =g_{p}[m]-d_{p}[A] .
\end{aligned}
$$

The parameters $d_{m}$ and $d_{p}\left(\mathrm{sec}^{-1}\right)$ are the degradation rates of the mRNAs and proteins, respectively. The trascription rate is given by $g_{m}\left(\mathrm{sec}^{-1}\right)$. The rate in which each mRNA is translated into proteins is given by $g_{p}\left(\mathrm{sec}^{-1}\right)$. Since $A$ proteins negatively regulate their own synthesis, the transcription rate is reduced by a factor of $1 /\left(1+k[A]^{n}\right)$. This factor is called the Hill-function. In this expression, the parameter $k$ quantifies the regulation strength (determined by the affinity between the repressor and the promoter site). The exponent $n$ is called the Hill-coefficient. In general, Hill-function models can be derived from more complete rate equation models. In this case, $n$ is expected to take only integer values. In fact, $n$ represents the number of copies of the transcription factor, that are required to be bound simultaneously to the promoter in order to perform the regulation. The case of $n>1$ is often referred to as cooperative binding. In this paper we consider only integer values of $n$. However, similar models may also be used to fit empirical data. In this case, $n$ is simply a fitting parameter which may take non-integer values.

To simplify the analysis of genetic circuits, the mRNA level is often ignored and the transcription and translation processes are regarded as a single step of protein synthesis [25]. In this case, the effective production rate of proteins is given by $g=g_{p} g_{m} / d_{m}$. The 
Michaelis-Menten equations are reduced to

$$
[\dot{A}]=\frac{g}{1+k[A]^{n}}-d[A],
$$

where $d=d_{p}$. Ignoring the mRNA level is typically justified under steady state conditions. However, in the analysis of systems that are away from steady state due to external signals, or those that exhibit oscillations or large fluctuations the mRNA level should be included. The Michaelis-Menten equations for the autorepressor exhibit a single steady state solution for the concentration of $A$ proteins. In case that $n=1$, it is given by

$$
[A]=\frac{-1+\sqrt{1+4 k g / d}}{2 k} \text {. }
$$

This solution is found to be stable for any choice of the parameters.

2.2. Extended set of rate equations. In the Michaelis-Menten equations the negative regulation process is described by the Hill-function. This description is rather crude and incomplete. In order to model the regulation process in greater detail we present below a more complete rate equation model [12]. In this model, we account separably for the populations of free and bound proteins. The bound $A$ proteins are denoted by $r$ and their concentration is given by $[r]$.

Consider an autorepressor gene $a$, encoded on the chromosome, which exhibits no cooperative binding. In this case the number of bound repressors is in the range $0 \leq$ $[r] \leq 1$. The concentration, $[r]$, can also be considered as the fraction of time in which the promoter is occupied by a bound repressor and the transcription process is suppressed. Therefore the transcription rate is reduced by a factor of $(1-[r])$. Ignoring the mRNA level, the extended set of rate equations takes the form [12]

$$
\begin{aligned}
{[\dot{A}] } & =g(1-[r])-d[A]-\alpha_{0}[A](1-[r])+\alpha_{1}[r], \\
{[\dot{r}] } & =\alpha_{0}[A](1-[r])-\alpha_{1}[r],
\end{aligned}
$$

where the parameter $\alpha_{0}\left(\mathrm{sec}^{-1}\right)$ is the binding rate of the repressors to the promoter site and $\alpha_{1}\left(\mathrm{sec}^{-1}\right)$ is the their unbinding rate. In the limit in which the binding and unbinding processes are much faster than other processes in the system (namely $\alpha_{0}, \alpha_{1} \gg d, g$ ), these equations can be reduced to the Michaelis-Menten form. In this limit, the relaxation time of the concentration $[r]$ is much shorter than other relaxation times in the system. Therefore, one can take the time derivative of $[r]$ to zero, even if the system is away from steady state. This brings the rate equations to the Michaelis-Menten form [Eq. (2)] with $n=1$ and $k=\alpha_{0} / \alpha_{1}$. Therefore, Eqs. (4) have the same steady state solution for the protein $A$ as the Michaelis-Menten equation (2). However, the dynamics leading to steady state may differ between the two equations. Furthermore, the extended rate equation model exhibits more flexibility in the sense that it is much easier to insert additional features into Eqs. (4) than into Eq. (2). For example, it is possible to introduce degradation of bound repressors by adding the term $-d_{r}[r]$ to the equation for $[\dot{r}]$ in Eqs. (4).

2.3. Stochastic analysis. Transcription factors and other proteins, as well as their mRNAs in a cell, often appear in low concentrations $[9,17,8]$. In this case, fluctuations in the 
copy numbers of these molecules may play an important role in the dynamics of genetic networks. To obtain a better description of thse systems, one should take into account the discrete nature of the molecules rather than using continuous concentrations. Moreover, even in case that some transcription factor appears in a high concentration, the regulation is performed by a small number of copies that are bound to the promoter site. The fluctuations in the number of bound transcription factors give rise to large temporal variations in the transcription rate of the regulated gene.

In order to account for fluctuations in genetic networks, stochastic methods are required, such as the master equation or Monte Carlo simulations [1, 10, 16, 20, 23]. The master equation is expressed in terms of the probability distribution $P\left(N_{A}, N_{r}\right)$. This is the probability for a cell to include $N_{A}=0,1,2, \ldots$ free copies of protein $A$ and $N_{r}=0,1$ copies of the same protein, which are bound to the promoter. The master equation accounts for the temporal variations in the probability distribution. For the autorepressor, it takes the form [12]

$$
\begin{aligned}
\dot{P}\left(N_{A}, N_{r}\right) & =g \delta_{N_{r}, 0}\left[P\left(N_{A}-1, N_{r}\right)-P\left(N_{A}, N_{r}\right)\right] \\
& +d\left[\left(N_{A}+1\right) P\left(N_{A}+1, N_{r}\right)-N_{A} P\left(N_{A}, N_{r}\right)\right] \\
& +\alpha_{0}\left[\delta_{N_{r}, 1}\left(N_{A}+1\right) P\left(N_{A}+1, N_{r}-1\right)-\delta_{N_{r}, 0} N_{A} P\left(N_{A}, N_{r}\right)\right] \\
& +\alpha_{1}\left[\delta_{N_{r}, 0} P\left(N_{A}-1, N_{r}+1\right)-\delta_{N_{r}, 1} P\left(N_{A}, N_{r}\right)\right],
\end{aligned}
$$

where the $g$ term accounts for the production of proteins and the $d$ term accounts for their degradation. The $\alpha_{0}\left(\alpha_{1}\right)$ terms describe the binding (unbinding) of proteins to (from) the promoter site.

In numerical integration, the master equation must be truncated in order to keep the number of equations finite. This is done by setting a suitable upper cutoff, $N_{\mathrm{A}}^{\max }$, on the population size of the free proteins. In order to maintain the accuracy of the calculations, the cutoff should be chosen such that the probability of population sizes beyond it will be sufficiently small. The master equation exhibits a single steady state solution, characterized by $\dot{P}\left(N_{A}, N_{r}\right)=0$ for all $N_{A}$ and $N_{r}$. This solution is always stable [27]. The average concentration of free $A$ proteins can be obtained from

$$
\left\langle N_{\mathrm{A}}\right\rangle=\sum_{N_{\mathrm{A}}=0}^{N_{\mathrm{A}}^{\max }} \sum_{N_{\mathrm{r}}=0}^{1} N_{\mathrm{A}} P\left(N_{A}, N_{r}\right) .
$$

Other properties of the distribution, such as the variance, can be obtained from the calculation of higher moments.

Another useful approach to the study of stochastic dynamics is provided by Monte Carlo methods $[7,9,16]$. The simulation dynamics is Markovian. At each instant, the next process to take place is chosen randomly from all the possible processes, where each process is assigned with a suitable weight, proportional to its rate. The elapsed time is then updated accordingly. Unlike the master equation, which accounts for the entire distribution, Monte Carlo simulations follow the temporal variations in protein concentrations in a single cell.

In Fig. 2(a) we present the concentration of $A$ proteins vs. time, obtained from the rate equations [Eq. (4)] and from Monte Carlo simulations. In Fig. 2(b) we show the 



Fig. 2. Results for the autorepressor circuit. (a) The concentration of $A$ proteins vs. time, obtained from the rate equations and from Monte Carlo simulations. The rate equation results quickly reach a steady state. The Monte Carlo results fluctuate around this steady state. (b) The steady state probability distribution $P\left(N_{A}\right)$ for a cell to contain $N_{A}$ copies of protein $A$, obtained from the master equation. The parameters used are $g=0.05, d=0.001, \alpha_{0}=0.01$, $\alpha_{1}=0.01$ and $d_{r}=0\left(\sec ^{-1}\right)$.

probability distribution $P\left(N_{\mathrm{A}}\right.$ obtained from the master equation under steady state conditions.

3. The genetic switch. The genetic toggle switch consists of two proteins, $A$ and $B$, which negatively regulate each other at the transcriptional level [Fig. 1(b)]. This architecture may lead to two steady states, one dominated by $A$ proteins and the other dominated by $B$ proteins. When the population of $A$ proteins is much larger than that of $B$ proteins, the $A$ proteins tend to suppress the production of $B$ proteins. Under these conditions, the production of $A$ proteins is enhanced, because the declining concentration of $B$ proteins is not sufficient to suppress it. Therefore, the system approaches a state rich in $A$ proteins and poor in $B$ proteins. Similarly, the system may approach a state rich in $B$ proteins and poor in $A$ proteins. Transitions between the two states may take place in response to a suitable external signal. Spontaneous transitions, due to random fluctuations, are also possible. To qualify as a switch, the system should exhibit bistability. In the deterministic description, bistability is defined as the existence of two stable steady state solutions of the rate equations. This description does not account for the possibility of spontaneous transitions between the two states. In the stochastic description, spontaneous transitions are taken into account. Therefore, the condition for bistability is that the rate of spontaneous transitions (due to random fluctuations rather than an external signal) is much lower than the rates of all other relevant processes in the system. 
Genetic switch systems exist in nature, and give rise to different cell behaviors in different situations. A notable example is the phage $\lambda$ switch [24]. This switch appears in $\lambda$ phages, which infect $E$. coli bacteria and can exist in two exclusive states, one called lysogeny and the other called lysis. In addition to natural switches, a synthetic switch was constructed and studied in E. coli [6]. Numerous studies, using rate equations, have concluded that cooperative binding is a necessary condition for the emergence of bistability $[3,6,28,29,30]$. Stochastic analysis reveals the reason to this fact. For a switch without cooperative binding, three peaks are obtained in the probability distribution function. These peaks corresponds to three possible states for the system: one in which $A$ is highly expressed, a second in which $B$ is highly expressed and a third in which both proteins are suppressed (a 'deadlock' situation) [13, 14]. Monte Carlo simulations show rapid transitions between these three states. The possibility of simultaneous suppression of both proteins, prevents the system from functioning as a switch. It causes the system to exhibit three states of limited stability instead of the two stable states that are desired.

It is found that in switch systems in which the $A$ and $B$ repressors exhibit cooperative binding, the deadlock situation is removed and bistability emerges. This can be explained as follows. The deadlock situation results from a simultaneous binding of $A$ and $B$ repressors to the corresponding promoter sites. Without cooperative binding, it is sufficient for the minority protein to recruit a single copy that will bind and suppress the production of the dominant protein. In the case of cooperative binding (for example, with $n=2$ ) the minority protein needs to recruit two copies that will bind simultaneously in order to suppress the production of the dominant protein. This is much less likely. Therefore, cooperative binding induces bistability and enables the system to function as a switch.

Apart from cooperative binding, there are several other mechanisms that may stabilize bistability in genetic switch systems. The most obvious is the exclusive switch, where there is an overlap between the promoters of $A$ and $B$, leaving no room for both to be occupied simultaneously. Such situations are encountered in nature, for example, in the lysis-lysogeny switch of phage $\lambda$ [24]. It was shown that in the presence of cooperative binding, the exclusive binding of $A$ and $B$ repressors enhances the stability of the genetic switch [29]. This is because in exclusive binding the access of the minority specie to the promoter site is blocked by the dominant specie. For the exclusive switch without cooperative binding, rate equations exhibit only a single steady state solution, namely, the system is not a switch in the deterministic framework. However, in the stochastic framework the system exhibits bistability and functions as a switch. The distribution $P\left(N_{A}, N_{B}\right)$ exhibits two peaks, one dominated by $A$ proteins and the other dominated by $B$ proteins. The exclusive binding prevents the possibility of a deadlock situation.

In addition to the exclusive switch, there exist other variants of the genetic switch circuit (we focus here on systems without cooperative binding). Consider a switch in which not only free proteins, but also bound proteins experience degradation. Boundrepressor degradation tends to prevent the deadlock situation in which both $A$ and $B$ repressors are bound simultaneously. This is due to the fact that degradation removes the bound repressor from the system, unlike unbinding, where the resulting free repressor may quickly bind again. It turns out that degradation of bound repressors induces bistability not only in the stochastic framework but also at the level of deterministic rate equations. 


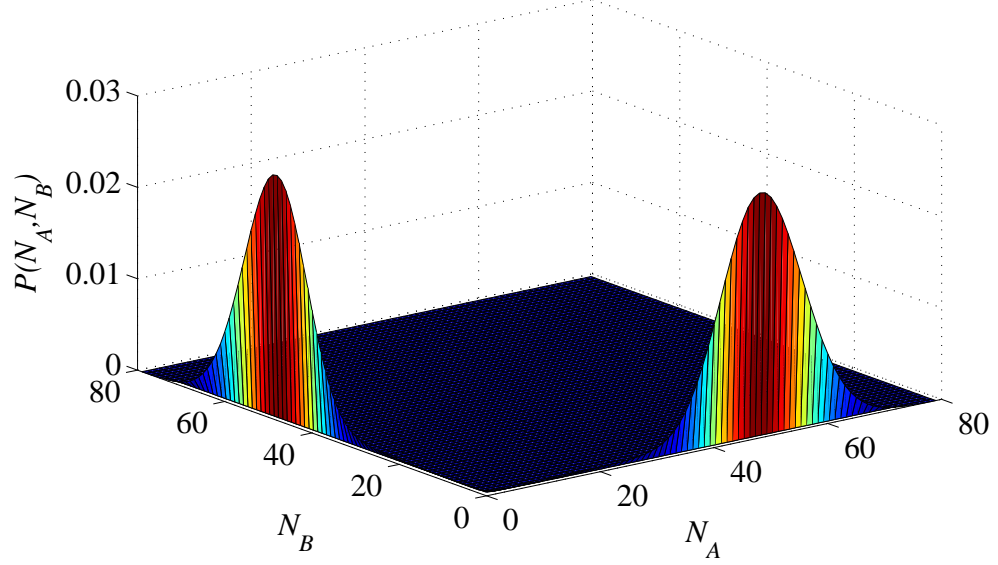

Fig. 3. The probability distribution $P\left(N_{\mathrm{A}}, N_{\mathrm{B}}\right)$ of the concentrations of $A$ and $B$ proteins, for the switch with degradation of bound repressors, obtained from the master equation. Two sharp peaks are observed, one dominated by $A$ proteins and the other dominated by $B$ proteins. The peaks are sharp and are separated by a region with vanishing probabilities. As a result, the transition rate between the two peaks is low and the switch is stable.

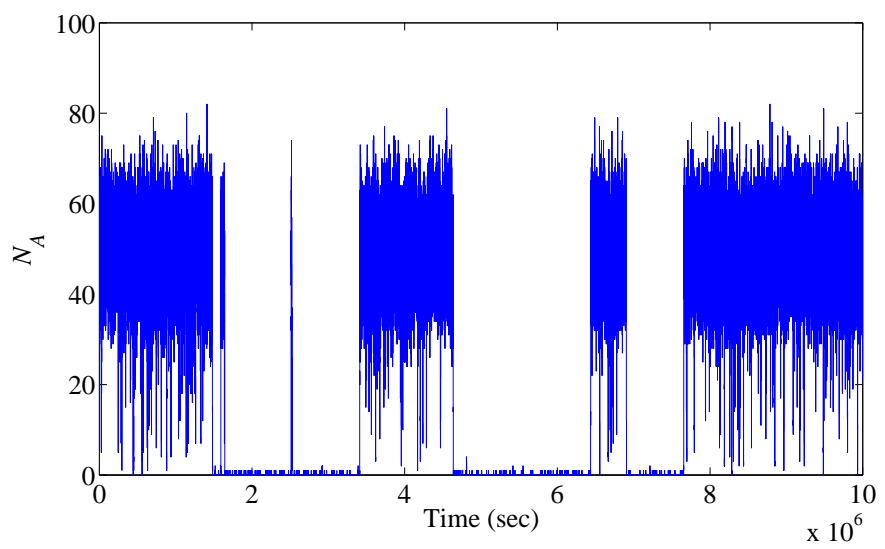

Fig. 4. The concentration, $N_{A}$, of $A$ proteins vs. time, obtained from Monte Carlo simulations for the switch with degradation of bound repressors. The two states are clearly observed: one in which $N_{A}$ fluctuates around 50, and another in which it is nearly zero. Transitions between these states occur at an average rate of one transition every $\sim 10^{6} \mathrm{sec}$ (about 10 days).

In Fig. 3 we present the probability distribution $P\left(N_{\mathrm{A}}, N_{\mathrm{B}}\right)$ of the concentrations of $A$ and $B$ proteins, for the switch with degradation of bound repressors. Two sharp peaks, well separated from each other are observed, illuminating the bistable nature of the systems. The parameters used in Fig. 3 and in the rest of the paper are: $g=0.15$, $d=0.003, \alpha_{0}=0.5, \alpha_{1}=0.01$ and $d_{r}=0.003\left(\mathrm{sec}^{-1}\right)$.

In Fig. 4 we present the temporal variations of the concentration, $N_{A}$, of $A$ proteins, obtained from Monte Carlo simulations for the switch with degradation of bound repressors. Two states are clearly observed: one in which $N_{A}$ is dominant and another state 


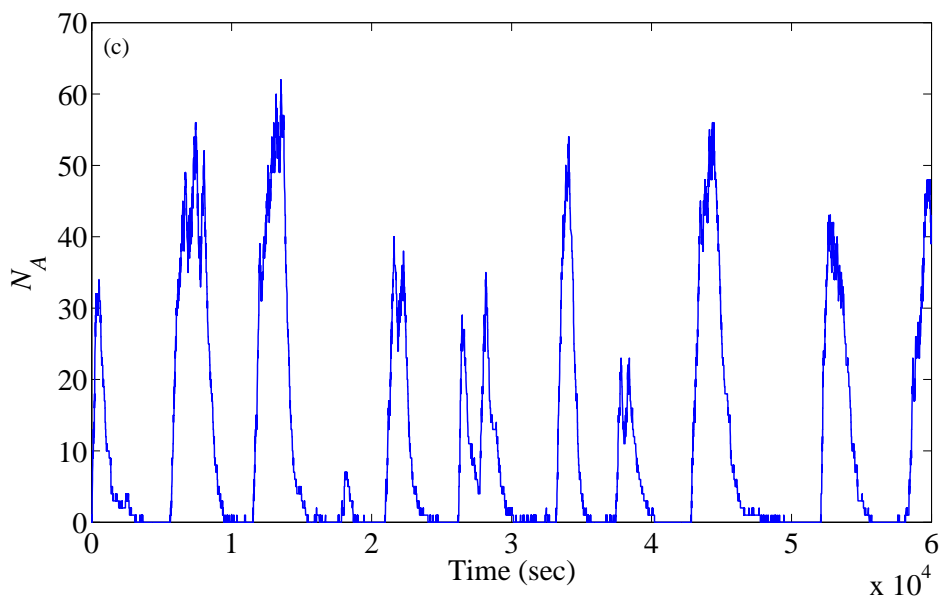

Fig. 5. The concentration, $N_{A}$, of $A$ proteins vs. time, obtained from Monte Carlo simulations of the repressilator circuit. The oscillations are noisy. Their period and amplitude vary from cycle to cycle.

in which it is suppressed. Note that in spite of the very large fluctuations, the switch is stable and the average time between spontaneous transitions is about 10 days.

Another variant of the genetic switch, which exhibits bistability even at the level of rate equations, involves protein-protein interactions, where $A$ and $B$ proteins bind to each other and form a complex that does not function as a transcription factor. This additional process contributes to the stability of the switch because in such 'pair annihilation' processes the minority protein is affected more strongly. It is thus less likely to bind and suppress the production of the dominant protein.

4. The repressilator. The repressilator circuit consists of three transcription factors, $A, B$ and $C$, which negatively regulate each other's synthesis in a cyclic manner [Fig. 1(c)]. This circuit was synthetically constructed on plasmids in E. coli and was found to exhibit oscillations in the concentrations of the three transcription factors. To understand the origin of these oscillations, consider a situation in which the number of $A$ proteins is large. In this case it is likely that one of the $A$ proteins will bind the to $b$ promoter and will repress the production of $B$ proteins. The reduced level of $B$ proteins will enable the gene $c$ to be fully expressed and the number of $C$ proteins will increase and will start to repress gene $a$. As a result, the number of $A$ proteins will decrease, and gene $b$ will be activated, completing a full cycle. The order of appearance of the dominant protein type in this cycle is $A \rightarrow C \rightarrow B \rightarrow A$.

From a theoretical point of view, oscillations in this system can be obtained (under some conditions) both in rate equations and in Monte Carlo simulations [Fig. 5]. The oscillations obtained from the rate equations are regular, forming a stable limit-cycle. The oscillations obtained from the Monte Carlo simulations are noisy and irregular. Moreover, the period and amplitude differ significantly between the rate equations and the Monte Carlo simulations [15]. 
The repressilator system was constructed synthetically on plasmids. When the number of plasmids in a cell is small, the numbers of promoter sites and bound transcription factors are also small. As a result, one expects large fluctuations in the transcription rates regulated by these transcription factors. In this case stochastic methods are required. However, when the number of plasmids is large, fluctuations are reduced and the rate equations become applicable. Therefore, by gradually changing the number of plasmids in the cell one can explore the cross-over from the stochastic regime, where the oscillations are noisy, to the deterministic regime, where the oscillations are regular.

5. Summary. We have presented deterministic and stochastic analysis of three simple genetic circuits which involve transcriptional regulation and feedback: the autorepressor, the switch and the repressilator. Such systems can be simulated using rate equations, that account for the concentrations of the mRNAs and proteins produced by these genes. Rate equations are suitable when these concentrations are large and fluctuations are negligible. However, when some of the transcription factors and their binding sites in a cell appear in low copy numbers, fluctuations become important and the rate equations fail. In this case stochastic methods such as the master equation or Monte Carlo simulations are required. We have shown that fluctuations give rise to quantitative and qualitative changes in the dynamics of the systems. In particular, we demonstrated the fluctuations-induced bistability in the exclusive switch and the noisy oscillations obtained in the repressilator circuit.

\section{References}

[1] A. Arkin, J. Ross and H. H. McAdams, Stochastic kinetic analysis of developmental pathway bifurcation in $\lambda$-infected E. coli cells, Genetics 149 (1998), 1633.

[2] A. Becskei and L. Serrano, Engineering stability in gene networks by autoregulation, Nature 405 (2000), 590.

[3] J. L. Cherry and F. R. Adler, How to make a biological switch, J. Theor. Biol. 203 (2000), 117.

[4] M. B. Elowitz and S. Leibler, A synthetic oscillatory network of transcriptional regulators, Nature 403 (2000), 335.

[5] M. B. Elowitz, A. J. Levine, E. D. Siggia and P. S. Swain, Stochastic gene expression in a single cell, Science 297 (2002), 1183.

[6] T. S. Gardner, C. R. Cantor and J. J. Collins, Construction of a genetic toggle switch in E. coli, Nature 403 (2000), 339.

[7] M. A. Gibson and J. Bruck, Efficient exact stochastic simulation of chemical systems with many species and many channels, J. Phys. Chem. 104 (2000), 1876.

[8] M. A. Gibson and E. Mjolsness, Modeling the activity of single genes, in: J. M. Bower and H. Bolouri, (eds.), Computational Modeling of Genetic and Biochemical Networks, MIT Press, Cambridge, MA, 2000, 1-48.

[9] D. T. Gillespie, Exact stochastic simulation of coupled chemical reactions, J. Phys. Chem. 81 (1977), 2340.

[10] T. B. Kepler and T. C. Elston, Stochasticity in transcriptional regulation: origins, consequences, and mathematical modeling representations, Biophysical Journal 81 (2001), 3116. 
[11] P. Laslo et al., Multilineage transcriptional priming and determination of alternate hematopoetic cell fates, Cell 126 (2006), 755.

[12] A. Lipshtat, H. B. Perets, N. Q. Balaban and O. Biham, Modeling of negative autoregulated genetic networks in single cells, Gene 347 (2005), 265.

[13] A. Lipshtat, A. Loinger, N.Q. Balaban and O. Biham, Genetic toggle switch without cooperative binding, Phys. Rev. Lett. 96 (2006), 188101.

[14] A. Loinger, A. Lipshtat, N. Q. Balaban and O. Biham, Stochastic simulations of genetic switch systems, Phys. Rev. E 75 (2007), 021904.

[15] A. Loinger and O. Biham, Stochastic simulations of the repressilator circuit, submitted to Phys. Rev. E (2007).

[16] H.H. McAdams and A. Arkin, Stochastic mechanisms in gene expression, Proc. Natl. Acad. Sci. USA 94 (1997), 814.

[17] H. H. McAdams and A. Arkin, It's a noisy business! Genetic regulation at the nanomolar scale, Trends Genet. 15 (1999), 65.

[18] R. Milo, S. Shen-Orr, S. Itzkovitz, N. Kashtan, D. Chklovskii and U. Alon, Network motifs: simple building blocks of complex networks, Science 298 (2002), 824.

[19] E. M. Ozbudak, M. Thattai, H. N. Lim, B. I. Shraiman and A. van Oudenaarden, Multistability in the lactose utilization network of Escherichia coli, Nature 427 (2004), 737.

[20] J. Paulsson and M. Ehrenberg, Random signal fluctuations can reduce random fluctuations in regulated components of chemical regulatory networks, Phys. Rev. Lett. 84 (2000), 5447.

[21] J. Paulsson, Stochastic focusing: fluctuation-enhanced sensitivity of intracellular regulation, Proc. Natl. Acad. Sci. USA 97 (2000), 7148.

[22] J. Paulsson, Multileveled selection on plasmid replication, Genetics 161 (2002), 1373.

[23] J. Paulsson, Summing up the noise in gene networks, Nature 427 (2004), 415.

[24] M. Ptashne, A Genetic Switch: Phage $\lambda$ and Higher Organisms, 2nd edition, Cell Press and Blackwell Scientific Publications, Cambridge, MA, 1992.

[25] N. Rosenfeld, M. B. Elowitz and U. Alon, Negative autoregulation speeds the response times of transcription networks, J. Mol. Biol. 323 (2002), 785.

[26] P. S. Swain, M. B. Elowitz and E. D. Siggia, Intrinsic and extrinsic contributions to stochasticity in gene expression, Proc. Natl. Acad. Sci. USA 99 (2002), 12795.

[27] N. G. van Kampen, Stochastic Processes in Physics and Chemistry, North-Holland, 1992.

[28] A. M. Walczak, M. Sasai, and P. Wolynes, Self consistent proteomic field theory of stochastic gene switches, Biophysical Journal 88, (2005), 828.

[29] P. B. Warren and P. R. ten Wolde, Enhancement of the stability of genetic switches by overlapping upstream regulatory domains, Phys. Rev. Lett. 92 (2004), 128101.

[30] P. B. Warren and P. R. ten Wolde, Chemical models of genetic toggle switches, J. Phys. Chem. B 109 (2005), 6812. 